\title{
Time density curve of dynamic contrast-enhanced computed tomography correlates with histological characteristics of pancreatic cancer
}

\author{
SHINTARO GOTO $^{1}$, HIROKO SEINO ${ }^{2}$, TADASHI YOSHIZAWA ${ }^{1}$, SATOKO MOROHASHI $^{1}$, \\ KEINOSUKE ISHIDO $^{3}$, KENICHI HAKAMADA ${ }^{3}$ and HIROSHI KIJIMA ${ }^{1}$ \\ ${ }^{1}$ Department of Pathology and Bioscience, Hirosaki University Graduate School of Medicine, Hirosaki, \\ Aomori 036-8562; ${ }^{2}$ Department of Radiology, Aomori National Hospital, Namioka, Aomori 038-1331; \\ ${ }^{3}$ Department of Gastroenterological Surgery, Hirosaki University Graduate School of Medicine, \\ Hirosaki, Aomori 036-8562, Japan
}

Received July 22, 2020; Accepted January 14, 2021

DOI: $10.3892 / \mathrm{ol} .2021 .12537$

\begin{abstract}
Pancreatic ductal adenocarcinoma (PDAC) is characterized by an infiltrative growth pattern with intense desmoplastic stroma comprised of cancer-associated fibroblasts (CAFs). Additionally, the histological characteristics are considered to play a vital role in the poor prognosis of PDAC. However, the density of cancer cells, degree of desmoplasia and vascular proliferation varies in individual cases. We hypothesized that preoperative radiological images would reflect histological characteristics, such as cancer cell density, CAF density and microvessel density. To clarify the association between the histological characteristics and radiological images of PDAC, the cancer cell density, CAF density and microvessel density from surgical specimens were measured with immunostaining, and the time density curve of dynamic contrast-enhanced computed tomography (CECT) was analyzed. Overall, the initial slope between non-enhanced and arterial phases was correlated with microvessel density, and the second slope between arterial and portal phases was correlated with CAF and cancer cell densities. In conclusion, the present study suggested the possibility of estimating cancer
\end{abstract}

Correspondence to: Dr Tadashi Yoshizawa, Department of Pathology and Bioscience, Hirosaki University Graduate School of Medicine, 5 Zaifu-cho, Hirosaki, Aomori 036-8562, Japan

E-mail: tyoshi@hirosaki-u.ac.jp

Abbreviations: aSMA, $\alpha$-smooth muscle actin; CAF, cancer-associated fibroblasts; CD31, cluster of differentiation 31; CECT, contrast-enhanced computed tomography; CEMRI, contrast-enhanced magnetic resonance imaging; NAC, neoadjuvant chemotherapy; PDAC, pancreatic ductal adenocarcinoma; ROI, region of interest; TDC, time density curve; TIC, time intensity curve

Key words: CAFs, microvessel density, pancreatic cancer, PDAC, contrast-enhanced computed tomography, TDC cell, CAF and microvessel densities using the TDC of dynamic CECT.

\section{Introduction}

Pancreatic cancer is one of the most malignant tumors of the digestive system globally (1). The Global Cancer Observatory (GLOBOCAN) estimated pancreatic cancer caused $>432,242$ deaths per year, accounting for $4.5 \%$ of all deaths worldwide in 2018 (2). Inspite of the achievements in diagnosis and management of pancreatic cancer, the disease-free overall 5-year survival rate is $\sim 6 \%$ (2-9\%) (3). Histologically, pancreatic ductal adenocarcinoma (PDAC) is the most common histological type. The most typical histological feature of PDAC is an infiltrative growth pattern with intensive desmoplastic stroma that is comprised of cancer-associated fibroblasts (CAFs) characterized by $\alpha$-smooth muscle actin ( $\alpha$ SMA) expression (4). Several studies have suggested that CAFs promote cancer progression through various intercellular communications, such as transforming growth factor $b$ (TGF-b) signaling, stimulating angiogenesis etc. (5-11).

In fact, the growth pattern of PDAC and degree of stromal desmoplasia varies in individual cases. The infiltrative growth pattern with abundant fibrous stroma is indeed the most common type; however, some patients show solid growth. We hypothesized that these histological differences in PDAC would be associated with the preoperative image, such as that obtained via dynamic contrast-enhanced computed tomography (CECT). This hypothesis was inspired by the following studies of contrast-enhanced magnetic resonance imaging (CEMRI). In studies on breast cancer, the time intensity curve (TIC) of CEMRI is considered to reflect tumor grade (12-14). The TIC forms a line chart that represents contrast enhancement changes dependent on time. The TIC consists of early and delayed phases; the early phase is described as slow, medium or rapid depending on the curve steepness, whereas the delayed phase occurs after the peak enhancement and is characterized as persistent if it continues to increase, as a plateau if it levels off and as washout if it decreases. Persistent 
kinetics are generally aligned more with benign lesions, whereas washout kinetics are generally suspected to be malignant and plateau kinetics are considered indeterminate (14). In Hirosaki University Hospital (Aomori, Japan), patients with PDAC are routinely examined with dynamic CECT as part of a fixed protocol. The usefulness of dynamic CECT in the diagnostic imaging and staging of pancreatic cancer has been established. Typical PDAC appears as hypodense masses compared with adjacent normal pancreatic tissue and draws the time density curve (TDC) for CECT as TIC is drawn for CEMRI. Comparative analysis between histology and TIC has been established in breast cancer; however, to the best of our knowledge only 1 study focused on the association between imaging diagnosis and PDAC histology (15). Therefore, the present study investigated the association between the histological characteristics and TDC of PDAC.

\section{Materials and methods}

Patients. A total of 59 patients with PDAC without preoperative chemotherapy, who were surgically treated between January 2012 and June 2018, were investigated after obtaining each patient's informed consent for using their clinical records and pathological specimens at the Hirosaki University Hospital (Hirosaki, Japan). The patient cohort was comprised of 34 women and 25 men, with a median age of 68 years (range, 53-84 years), and with tumors located in the pancreatic head ( 23 cases), pancreatic body (27 cases) and pancreatic tail (9 cases). All patients underwent dynamic CECT, and none of them had a contrast media allergy or renal function problems that would prevent them from undergoing CECT. Operative procedures were determined according to the location of the primary tumors and tumor spread. Pancreatoduodenectomy was conducted in the 21 patients with pancreatic head cancer, and distal pancreatosplenectomy was conducted in the 36 patients with pancreatic body and tail cancer. A total of 2 patients with pancreatic head cancer underwent a total pancreatectomy due to tumor spread in the main duct of the pancreatic body. At the Hirosaki University Hospital, patients are administered S-1 (tegafur/gimeracil/oteracil) as standard postoperative chemotherapy for pancreatic cancer and the course is followed up. S-1 was administered orally twice daily at a dose of $80 \mathrm{mg} / \mathrm{m}^{2} /$ day for 4 weeks and 2 weeks rest. This cycle was repeated for half a year. In the present study, S-1 was administered to 55 patients. S-1 administration was discontinued in 4 cases due to adverse effects. Survival data were obtained from hospital medical charts. A total of 49 patients were surveyed, but 10 patients did not complete the follow-up survey. The median observation period was 27.8 months.

Histological analysis. All surgical specimens were fixed with $10 \%$ formalin at room temperature for $24 \mathrm{~h}$. In cases of pancreatic head tumors, surgical specimens were sliced at right angles to the common bile duct, whereas those in the pancreatic body or tail tumor were sliced at right angles to the main pancreatic duct. Tumors were embedded in paraffin, and $4-\mu \mathrm{M}$ sections were stained with hematoxylin and eosin (H\&E) for pathological evaluation. The sections were stained at room temperature with Mayer's hematoxylin for $20 \mathrm{~min}$ and with eosin for $5 \mathrm{~min}$. Pathological evaluations, including pathological T, $\mathrm{N}$ and $\mathrm{M}$ categories, and staging were conducted for all patients according to an up-to-date TNM classification from the Union for International Cancer Control (8th edition) (16). The PDAC differentiation was classified as well differentiated (G1), moderately differentiated (G2) and poorly differentiated (G3) according to the World Health Organization classification of tumors of the digestive system (5th edition) (17). If intratumoral heterogeneity (i.e., variation in the degree of differentiation) was present, a higher grade was assigned.

Immunostaining and histological image analyses. To measure the cancer cell, CAF and microvessel densities, the largest cross section of tumor was measured. Immunostaining of cytokeratin AE1/AE3, $\alpha$ SMA and cluster of differentiation 31 (CD31) was conducted. Cytokeratin AE1/AE3 is a pan-epithelial marker. Although there is no specific marker for CAFs, $\alpha$ SMA is widely used as a CAF marker in various human cancer types, such as lung cancer (18), cholangiocarcinoma (19), gastric cancer (20) and breast cancer (21). CD31 is a pan-vascular endothelial marker that is positive for both normal endothelial and tumor endothelial cells (22). For the immunostaining examination, sections on microslides were deparaffinized using the standard avidin-biotin-peroxidase complex method with automated immunostainer (Benchmark XT; Ventana Medical Systems, Inc.). In brief, deparaffinized slides were treated with tris-EDTA buffer $\left(\mathrm{pH} \mathrm{7.8)}\right.$ at $95^{\circ} \mathrm{C}$ for $44 \mathrm{~min}$. For blocking endogenous peroxides and protein, the slides were treated with $5 \%$ non-fat dry milk at $37^{\circ} \mathrm{C}$ for $15 \mathrm{~min}$. The slides were incubated with primary antibody for $60 \mathrm{~min}$ at room temperature. The clones and dilution ratios of primary antibodies were as follows: Cytokeratin AE1/AE3 (monoclonal mouse; clone AE1, AE3; 1:100; cat. no. 412811; Nichirei Bioscience, Inc.), $\alpha$ SMA (monoclonal mouse; clone 1A4; cat. no. M0851; 1:100; Dako; Agilent Technologies Inc.) and CD31 (monoclonal mouse; clone JC70A; cat. no. M0823; 1:40; Dako; Agilent Technologies Inc.). Reaction products were visualized by iVIEW DAB Detection kit including secondary antibody of biotin-binding goat anti mouse immunoglobulin G (IgG) (1:1,000; cat. no. 760-091; Ventana Medical Systems, Inc.; Roche Diagnostics). The largest slice was equally divided by field of view (field number was 22) using a microscope with a 4 X objective lens and a 10X eyepiece (Fig. 1A-C). BX53 light microscope (Olympus Corporation), CellSens software v.2.3 64 bit and DP74 digital camera (both Olympus Corporation) were used for capturing images. An attempt was made to exclude regions where the tumor and non-tumor tissue were mixed in the margins and to exclude the lumens of enlarged ducts from the measuring area, combining these images with observations from H\&E stained tissues. Whole images of the largest slice of tumor with immunostaining were captured (Fig. 1D-F), and immunostaining images were binarized using Image J software [Java 1.6.0_24 (64-bit); National Institutes of Health]. Binarized images showed immunostaining-positive and immunostaining-negative components as red and white (Fig. 1G-I). The area of immunostaining-positive components was measured as the pixel number, using thresholds with minimum and maximum values of 0 and 120 , respectively. Cancer cell density was measured as cytokeratin AE1/AE3-positive pixel number divided by the total area pixel 
Cytokeratin $\mathrm{AE} 1 / \mathrm{AE} 3$

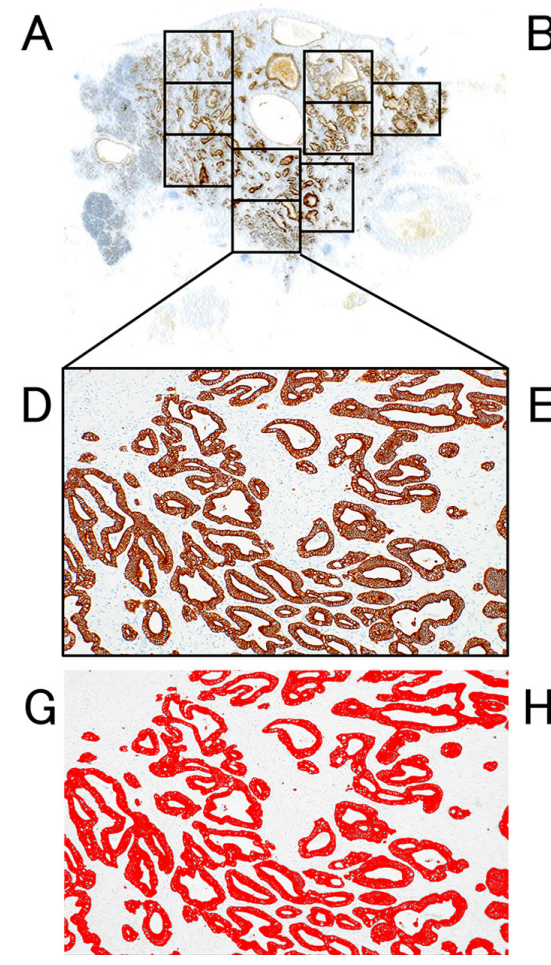

aSMA

B

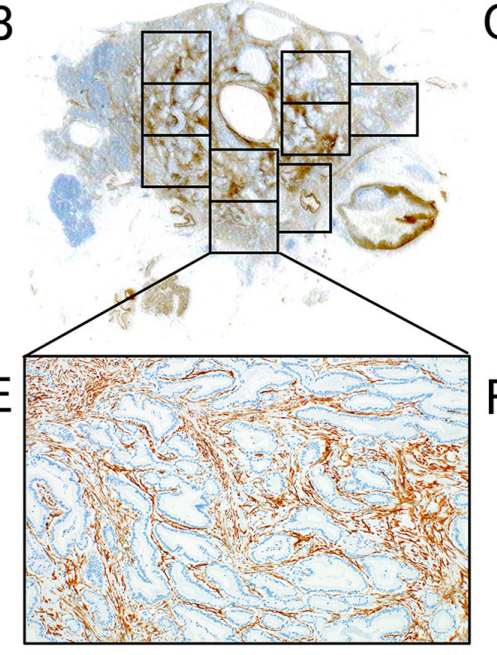

CD31

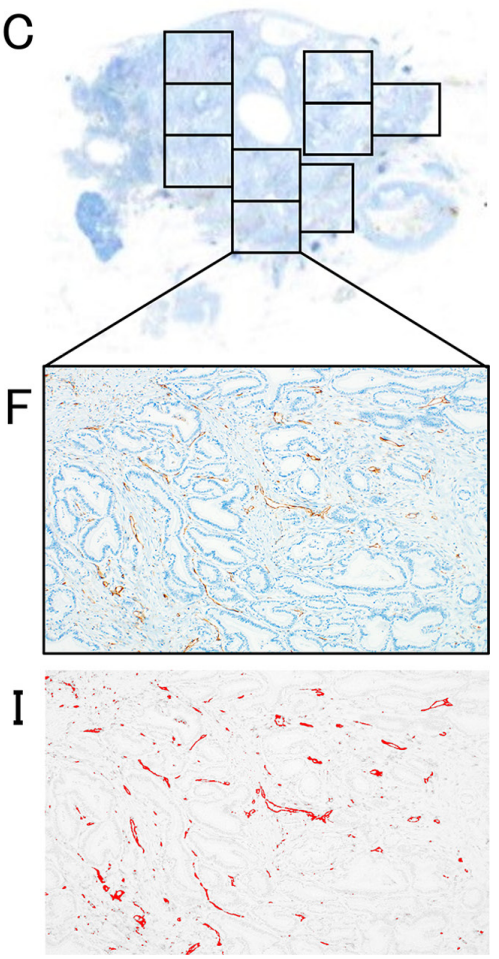

Figure 1. Measurements of the cancer cell density, CAF density and microvessel density using immunostaining. The largest slice of tumor stained with (A) cytokeratin AE1/AE3, (B) $\alpha$ SMA and (C) CD31 was equally divided by the fields of view of a microscope $4 \mathrm{X}$ objective lens and a 10X eyepiece, avoiding the non-tumor area and lumens of enlarged ducts. (D) AE1/AE3-stained cancer cells. (E) $\alpha$ SMA-stained CAFs and vascular smooth muscle. (F) CD31-stained endothelial cells of microvessels. (G-I) Binarized images of (D-F). Immunostaining-positive and immunostaining-negative components are red and white, respectively. Red areas were measured as pixel number. $\alpha$ SMA, $\alpha$-smooth muscle actin; CAF, cancer-associated fibroblast; CD31, cluster of differentiation 31 .

number, and CAF density was measured as the $\alpha$ SMA-positive pixel number minus the CD31-positive pixel number divided by the total stromal area pixel number, i.e., total area pixel number minus cancer cell pixel number. Microvessel density was measured as the CD31-positive pixel number divided by the total stromal area pixel number.

Radiological imaging analysis. Dynamic contrast-enhanced computed tomography (CECT) images were investigated using the fixed protocol applied to all patients with pancreatic cancer in the Hirosaki University Hospital. CT was conducted using a 64-detector row CT scanner (Discovery CT750 HD; GE Healthcare), using the following parameters: Detector configuration, $64 \times 0.625$; tube voltage, $120 \mathrm{kV}$; automatic tube current modulation; collimation, $40 \mathrm{~mm}$; tube rotation time, $0.5 \mathrm{sec}$; pitch, 0.8; field of view, $35 \times 35 \mathrm{~cm}$; image matrix, 512x512; and slice thickness, $5 \mathrm{~mm}$. After obtaining unenhanced images, a non-ionic contrast medium dose of $600 \mathrm{mgI} / \mathrm{kg}$ body weight with an iodine content of $300 \mathrm{mgI} / \mathrm{ml}$ (Iopamiron 300/370, Bayer Yakuhin Ltd.; Omnipaque 300, Daiichi-Sankyo Co., Ltd.; Iopromide 300/370, Fujifilm Toyama Chemical Co., Ltd.; Iomelon 350, Eisai Co., Ltd.; Optiray 320, Fuji Pharma Co., Ltd.) was intravenously injected within $30 \mathrm{sec}$, and scanning of the arterial, portal venous and equilibrium phases began at 35-40 sec, 60-70 sec and $180 \mathrm{sec}$ after initiating the contrast medium injection (Fig. 2A-D). To draw a TDC, Digital Imaging and Communications in Medicine data (EV insite $\mathrm{R}$ version 3.4.0.0 from PSP corporation or ShadeQuest/ViewR
V1.24 from Yokogawa Medical Solutions) were used. The primary tumor was indicated as the hypovascular area surrounded by a yellow dotted line. The region of interest (ROI) was indicated as the area surrounded by a solid yellow line (Fig. 2A-D). The ROI was drawn on the central area of the tumor and avoided tumor margins, the vascular structure and artificial materials such as stents. The ROI was drawn on the central area of the tumor, as the marginal area includes both tumor and non-tumor tissue on the H\&E slides. After measuring the CT number of ROIs in each phase, the TDC was created (Fig. 2E). T1 indicates the non-enhanced phase, T2 the arterial phase, T3 the portal phase and T4 the equilibrium phase. H1 is the CT number of the non-enhanced phase (T1), $\mathrm{H} 2$ is that of the arterial phase (T2), H3 is that of the portal phase (T3) and H4 is that of the equilibrium phase (T4). $\delta 1$ is the initial slope between non-enhanced and arterial phases, $\delta 2$ the second slope between arterial and portal phases and $\delta 3$ the third slope between portal and equilibrium phases.

Statistical analysis. The correlations between $\delta 1-3$ and the densities of cancer cells, CAFs and microvessels were statistically analyzed using Spearman's rank correlation coefficient. Correlation was defined as statistically significant if the $\rho$ value ( $r$ ) was calculated as $|r|>0.2$. Survival analyses were conducted using the Kaplan-Meier method to estimate event rates and the log-rank test for survival comparisons between patient groups. Cancer cell density, CAF density and microvessel density were divided into high and low groups based on respective median 


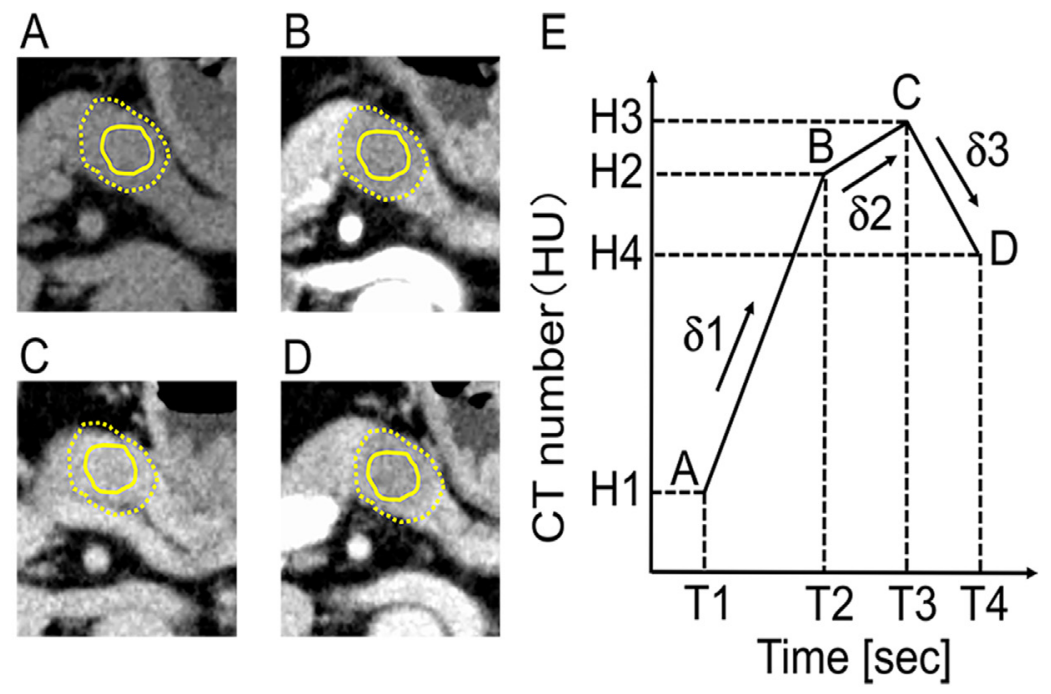

Figure 2. Measuring the CT number of ROI and drawing the TDC of PDAC. Dynamic CECT images of the pancreatic body cancer at the (A) non-enhanced, (B) arterial, (C) portal and (D) equilibrium phases. The margins of the tumor were represented by a yellow dotted line. The solid yellow line is the ROI. (E) Measuring the CT number of the ROI, the time intensity curve was drawn. T1, T2, T3 and T4 represent the times for (A-D), respectively. H1, H2, H3, and $\mathrm{H} 4$ represent the $\mathrm{CT}$ number of T1, T2, T3 and T4, respectively. $\delta 1$ was the initial slope between A and B. $\delta 2$ was the second slope between B and C. $\delta 3$ was the third slope between (C and D). CT, computed tomography; CECT, contrast-enhanced CT; PDAC, pancreatic ductal adenocarcinoma; ROI, region of interest; TDC, time density curve.

values. The median value of cancer cell density was $18.3 \%$, CAF density was $20.4 \%$, and microvessel density was $1.05 \%$. All statistical evaluations were conducted using R v.3.6.3 (http://www.r-project.org) and EZR v.1.41 (Saitama Medical Center, Jichi Medical University) software.

\section{Results}

Clinicopathological findings of PDAC. The pathological $\mathrm{T}$ category was represented as pT1c with 7 cases $(12 \%), \mathrm{pT} 2$ with 38 cases $(64 \%)$ and pT3 with 14 cases (24\%). Pathological $\mathrm{N}$ category was represented as pN0 with 20 cases $(34 \%)$, pN1 with 23 cases $(39 \%)$ and pN2 with 16 cases (27\%). Patients with distant metastasis were not included. Staging was recorded as stage IA in 4 cases (7\%), stage IB in 14 cases (24\%), stage IIA in 2 cases (3\%), stage IIB in 22 cases $(37 \%)$ and stage III in 17 cases (29\%) (Table I). Tumor differentiation was well differentiated (G1) in 4 cases (7\%), moderately differentiated (G2) in 42 cases $(71 \%)$ and poorly differentiated (G3) in 13 cases (22\%). The median cancer cell density was $18.3 \%$ (range, $5.0-41.7 \%$ ), the CAF density was $20.4 \%$ (range, $0.5-55.1 \%$ ) and the microvessel density was $1.05 \%$ (range, 0.09-0.40\%) (Table II).

TDC and histopathology of PDAC. Three combinations showed statistically significant correlations: $\delta 1$ was correlated with microvessel density $(\mathrm{r}=0.366, \mathrm{P}=0.004)$ and $\delta 2$ was correlated with both cancer cell density $(r=-0.280, P=0.032)$ and CAF density $(\mathrm{r}=-0.301, \mathrm{P}=0.020)$. Additionally, $\delta 3$ was not associated with either cancer cell density $(\mathrm{r}=-0.003$, $\mathrm{P}=0.979$ ) or CAF density ( $\mathrm{r}=0.234, \mathrm{P}=0.074$ ) (Fig. 3); $\delta 2$ was not associated with microvessel density $(\mathrm{r}=-0.080, \mathrm{P}=0.547)$ and $\delta 3$ was not associated with cancer cell density $(\mathrm{r}=-0.087$, $\mathrm{P}=0.510), \mathrm{CAF}$ density $(\mathrm{r}=0.037, \mathrm{P}=0.782)$ or microvessel density ( $\mathrm{r}=-0.005, \mathrm{P}=0.969)$ (Table III). Representative cases were shown in Fig. 4. The TDC of Case 1 showed a gentle upslope in $\delta 1$ and $\delta 2$ (Fig. 4). Histologically, Case 1 showed a solid growth pattern with high cancer cell density, high CAF density and low microvessel density (Fig. 4). On the other hand, the TDC of Case 2 showed a rapid upslope in $\delta 1$ and $\delta 2$ (Fig. 4). Histologically, Case 2 showed an infiltrative growth pattern with low cancer cell density, low CAF density and high microvessel density (Fig. 4).

Association between cancer cell density, CAF density and microvessel density. There were no significant correlations between cancer cell density and CAF density $(r=0.062$, $\mathrm{P}=0.645), \mathrm{CAF}$ density and microvessel density $(\mathrm{r}=0.240$, $\mathrm{P}=0.069)$, or cancer cell density and microvessel density $(\mathrm{r}=0.076, \mathrm{P}=0.572)$ (Table IV).

Prognostic analysis of PDAC. Differences in overall survival time between high and low cancer cell density groups $(\mathrm{P}=0.522)$, between high and low CAF density groups $(\mathrm{P}=0.573)$, and between high and low microvessel density groups ( $\mathrm{P}=0.871)$ were not significant (Fig. 5).

\section{Discussion}

The present study demonstrated the possibility that TDC allows us to predict the histological characteristics of PDAC. The initial slope between the non-enhanced phase and the arterial phase ( $\delta 1)$ was positively correlated with microvessel density, and the second slope between the arterial phase and the portal phase $(\delta 2)$ was negatively correlated with cancer cell density and CAF density. For example, the TDC with a high $\delta 1$ indicated high vascularization, and the TDC with a low $\delta 2$ indicated high cancer cell density and high CAF density. Tumors with a high $\delta 1$ and a low $\delta 2$ tended to display histological characteristics such as a solid growth pattern with a stroma containing many CD31-positive microvessels and dense $\alpha$ SMA-positive fibrosis. Thus, the shape of the TDC 
Table I. Clinical features of 59 patients with pancreatic ductal adenocarcinoma.

\begin{tabular}{lc}
\hline Clinical features & Value \\
\hline Mean age (range), years & $69.2(53-84)$ \\
Sex, n (\%) & \\
Female & $34(58)$ \\
Male & $25(42)$ \\
Location, n (\%) & \\
Pancreatic head & $23(39)$ \\
Pancreatic body & $27(46)$ \\
Pancreatic tail & $9(15)$ \\
Pathological T category, n (\%) & \\
pT0, pTis, pT1a, pT1b, n (\%) & \\
pT1c & $0(0)$ \\
pT2 & $7(12)$ \\
pT3 & $38(64)$ \\
pT4 & $14(24)$ \\
Pathological N category, n (\%) & $0(0)$ \\
pN0 & \\
pN1 & $20(34)$ \\
pN2 & $23(39)$ \\
M category, n (\%) & $16(27)$ \\
M0 & \\
M1 & $59(100)$ \\
Staging, n (\%) & $0(0)$ \\
Stage 0 & \\
Stage IA & $0(0)$ \\
Stage IB & $4(7)$ \\
Stage IIA & $14(24)$ \\
Stage IIB & $2(3)$ \\
Stage III & $22(37)$ \\
Stage IV & $17(29)$ \\
\hline & 0 \\
\hline
\end{tabular}

reflects the tumor growth pattern and characteristics of the tumor stroma.

First, the initial slope between the non-enhanced and arterial phases $(\delta 1)$ was found to be positively correlated with microvessel density. The steepness of $\delta 1$ represents microvessel proliferation in the cancer stroma. It has been shown that the attenuation value of the peak enhancement of the tumor and the peak enhancement value divided by time are positively correlated with the extent of tumor vascularity in lung cancer and renal cell carcinoma $(23,24)$. In a study of 36 patients with PDAC, Hattori et al (15) reported that when using conventional dynamic multidetector CT (MDCT), the ratios of enhanced values in the arterial phase to the tumor-aorta enhanced values were correlated with CD34-positive microvessel density, as counted in five hot spots at high magnification. The present results regarding $\delta 1$ and microvessel density were consistent with those of this previous study. Since $\delta 1$ represents data on changes in contrast agents entering the tumor tissue over time, it is more likely to be correlated with tumor vascularity. Although the difference in
Table II. Histological findings of pancreatic ductal adenocarcinoma.

\begin{tabular}{lcc}
\hline Histological findings & $\begin{array}{c}\text { Patients, } \\
\mathrm{n}(\%)\end{array}$ & $\begin{array}{c}\text { Median, } \% \\
\text { (range) }\end{array}$ \\
\hline Differentiation & & \\
G1 & $4(7)$ & \\
$\mathrm{G} 2$ & $42(71)$ & \\
$\mathrm{G} 3$ & $13(22)$ & \\
Cancer cell density, $\mathrm{n} \%$ & & $18.3(5.0-41.7)$ \\
$\mathrm{n}<10$ & $6(10)$ & \\
$10 \leq \mathrm{n}<20$ & $31(53)$ & \\
$20 \leq \mathrm{n}<30$ & $17(29)$ & \\
$30 \leq \mathrm{n}<40$ & $3(5)$ & \\
$\mathrm{n} \geq 40$ & $2(3)$ & \\
$\mathrm{CAF}$ density, $\mathrm{n} \%$ & & \\
$\mathrm{n}<10$ & $15(25)$ & \\
$10 \leq \mathrm{n}<20$ & $11(19)$ & \\
$20 \leq \mathrm{n}<30$ & $20(34)$ & \\
$30 \leq \mathrm{n}<40$ & $8(14)$ & \\
$\mathrm{n} \geq 40$ & $5(8)$ & \\
$\mathrm{Microvessel}$ density, $\mathrm{n} \%$ & & \\
$\mathrm{n}<1$ & $29(49)$ & \\
$1 \leq \mathrm{n}<2$ & $26(44)$ & \\
$2 \leq \mathrm{n}<3$ & $3(5)$ & \\
$\mathrm{n} \geq 3$ & $1(2)$ & \\
& &
\end{tabular}

CAF, cancer-associated fibroblast; G1, well differentiated; G2, moderately differentiated; G3, poorly differentiated; CAF, cancer-associated fibroblast.

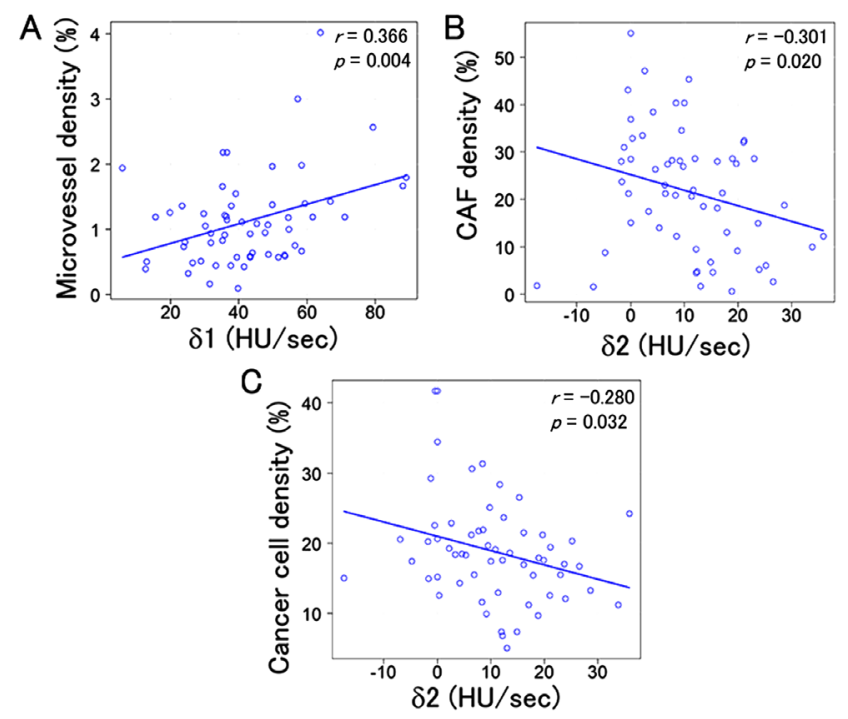

Figure 3. Correlation between the TDC and histopathology of pancreatic ductal adenocarcinoma. (A) $\delta 1$ was positively correlated with microvessel density ( $\mathrm{r}=0.366, \mathrm{P}=0.004)$. (B) $\delta 2$ was weakly negatively correlated with CAF density $(\mathrm{r}=-0.301, \mathrm{P}=0.020)$. (C) $\delta 2$ was weakly negatively correlated with cancer cell density $(\mathrm{r}=-0.280, \mathrm{P}=0.032)$. CAF, cancer-associated fibroblast; TDC, time density curve; $\delta 1$, the initial slope between the non-contrast and arterial phase of the TDC; $\delta 2$, the second slope between the arterial and portal phases of the TDC. 
Table III. Correlations between TDC and histological findings of pancreatic ductal adenocarcinoma.

\begin{tabular}{|c|c|c|c|c|c|c|}
\hline \multirow[b]{2}{*}{ Slope } & \multicolumn{2}{|c|}{ Cancer cell density } & \multicolumn{2}{|c|}{ CAF density } & \multicolumn{2}{|c|}{ Microvessel density } \\
\hline & $\mathrm{r}$ & P-value & $\mathrm{r}$ & P-value & $\mathrm{r}$ & P-value \\
\hline$\delta 1$ & -0.003 & 0.979 & 0.234 & 0.074 & 0.366 & 0.004 \\
\hline$\delta 2$ & -0.280 & 0.032 & -0.301 & 0.020 & -0.080 & 0.547 \\
\hline$\delta 3$ & -0.087 & 0.510 & 0.037 & 0.782 & -0.005 & 0.969 \\
\hline
\end{tabular}

CAF, cancer-associated fibroblast; TDC, time density curve; $\delta 1$, the initial slope between non-contrast and arterial phases of TDC; $\delta 2$, the second slope between arterial and portal phases of TDC; $\delta 3$, the third slope between portal and equilibrium phases of TDC.
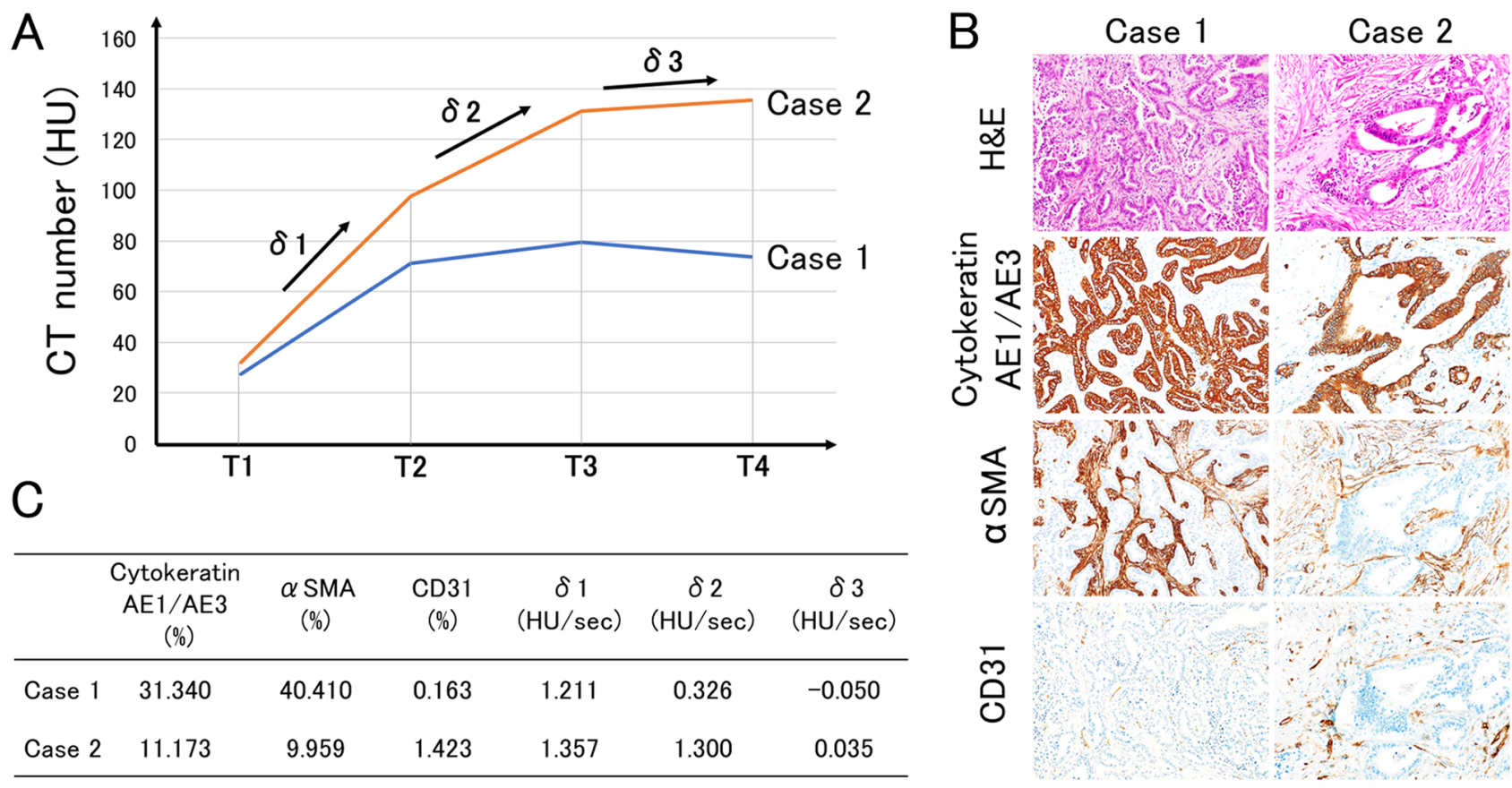

Figure 4. Representative cases. (A) TDCs of cases 1 and 2. Case 1 showed a gentle upslope in $\delta 1$ and $\delta 2$. Case 2 showed a rapid upslope in $\delta 1$ and $\delta 2$. (B) The histological characteristics of cases 1 and 2. Case 1 showed a solid growth pattern with high cancer cell density, high CAF density and low microvessel density. Case 2 showed an infiltrative growth pattern with low cancer cell density, low CAF density and high microvessel density. (C) The specifications of cases 1 and 2. Cytokeratin AE1/AE3 (\%) represents cancer cell density, $\alpha$ SMA (\%) represents CAF density and CD31 (\%) represents microvessel density. TDC, time density curve; T1, non-enhanced phase; T2, arterial phase; T3, portal phase; T4, equilibrium phase; $\delta 1$, the initial slope between T1 and T2; $\delta 2$, the second slope between T2 and T3; $\delta 3$, the third slope between T3 and T4; $\alpha$ SMA, $\alpha$-smooth muscle actin; CAF, cancer-associated fibroblast; CD31, cluster of differentiation 31 .

overall survival between the high and low microvessel density groups was not significant in the present study, angiogenesis has been considered a poor prognostic factor in many cancer types, such as breast (25-27), lung $(28,29)$, and colorectal (30) cancer. However, Katsuta et al (31) reported that the high expression of CD31 is associated with better overall survival time in patients with PDAC based on mRNA expression from RNA sequencing data (31). The study further elaborated that PDAC expressing high levels of CD31 has mature and stable vessels that supply anti-cancer immune cells. Thus, the association between tumor vascularity and prognosis in PDAC appears to be controversial.

Second, the second slope between the arterial and portal phases ( $\delta 2)$ was found to be negatively correlated with both cancer cell and CAF density. The gentleness or descent of $\delta 2$ is considered to represent high-density cancer cells and CAFs.
The study by Hattori et al (15) also reported that the extent of fibrosis was negatively correlated with the absolute value in the pancreatic phase of conventional dynamic MDCT, which is equivalent to the portal phase in the present study. The extent of fibrosis was scored according to the ratio of fibrosis in the tumor using Elastica van Gieson staining, with a score of 1-3 (15). The present study reinforced these results with quantitative data focusing on $\alpha$ SMA-positive fibroblasts. Generally, contrast agents have two-compartment pharmacokinetics with intravascular and extravascular-extracellular components. After an intravenous injection, contrast agents circulate and reach organs via the arterial system in the arterial phase. Thereafter, contrast agents leak from the microvessels to the pancreatic tissue in the portal phase. Lastly, contrast agents that accumulate in the pancreatic tissue return to the microvessels during the equilibrium phase. In the present 
Table IV. Correlations among histological findings of pancreatic ductal adenocarcinoma.

\begin{tabular}{|c|c|c|c|c|c|c|}
\hline \multirow[b]{2}{*}{ Histological findings } & \multicolumn{2}{|c|}{ Cancer cell density } & \multicolumn{2}{|c|}{ CAF density } & \multicolumn{2}{|c|}{ Microvessel density } \\
\hline & $\mathrm{r}$ & P-value & $\mathrm{r}$ & P-value & $\mathrm{r}$ & P-value \\
\hline Cancer cell density & - & - & 0.0618 & 0.645 & 0.0758 & 0.572 \\
\hline CAF density & - & - & - & - & 0.24 & 0.0691 \\
\hline Microvessel density & - & - & - & - & - & - \\
\hline
\end{tabular}

CAF, cancer-associated fibroblast.
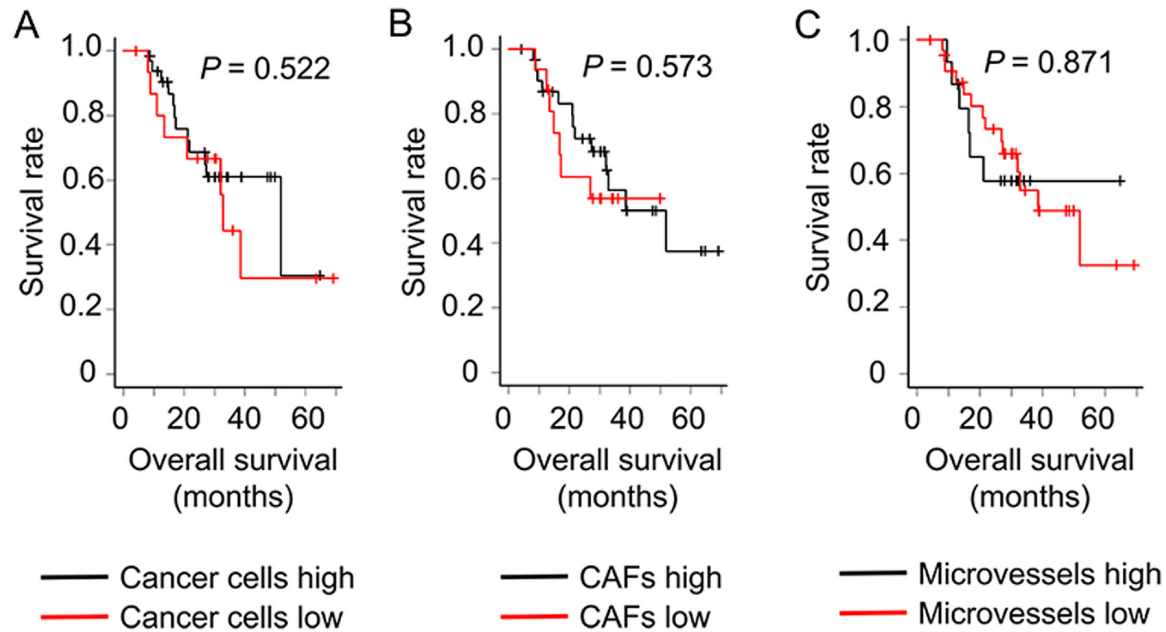

Figure 5. Associations between histopathology and overall survival of pancreatic ductal adenocarcinoma. The overall survival according to high/low (A) cancer cell, (B) CAF and (C) microvessel densities. The high and low groups were based on the median values. No significant associations were found between any histopathological features and overall survival. CAF, cancer-associated fibroblast.

study, a non-ionic iodine contrast agent was used, i.e., one not distributed into the cytoplasm of cancer cells and CAFs, but into the extracellular matrix (32). Therefore, cancer cells and CAFs work as obstacles to the spread of contrast agents, causing the negative correlations between $\delta 2$ and cancer cell and CAF densities. CAF proliferation has been considered a poor prognostic factor in numerous studies $(5-11,33,34)$. However, the difference in overall survival time between the high and low CAF density groups was not significant in the present study. A possible reason for the differences between the present study and previous studies is the non-specific immunostaining of $\alpha$ SMA. In the present study, the entire tumor area was measured, not just the tumor infiltrated areas, in order to compare tissue sections and radiological images. Furthermore, immunostaining was performed using an automated method with Image J. Therefore, it is possible that non-specific $\alpha \mathrm{SMA}$ immunostaining was detected. By contrast, a recent study revealed that there are two types of CAFs: Cancer-promoting CAFs and cancer-restraining CAFs (35). Cancer-promoting CAFs express $\alpha$ SMA (36) and cancer-restraining CAFs express Meflin, a glycosylphosphatidylinositol-anchored protein (37). Currently, in situ hybridization is recommended for detecting Meflin on tissue slides, as Meflin immunostaining often results in non-specific staining (37). Meflin is an attractive marker for future research to distinguish cancer-restraining CAFs from cancer-promoting CAFs.
Although the present study demonstrated a significant correlation between the TDC and histological characteristics, the correlation coefficients were small $(|r|=0.2-0.3)$. The following technical limitations are possible reasons for these small values. First, it was challenging to achieve complete exclusion of the marginal area, including both tumor and non-tumor tissues from the tissue assessment area, as cytokeratin AE1/AE3, $\alpha$ SMA and CD31 were stained on both the tumor and non-tumor tissues. On the other hand, it is important that the microenvironment of the tumor center is different from the tumor margin. The present study did not investigate the issue, and it is a challenge for the future. Second, it was difficult to achieve a complete match between the ROI of dynamic CECT and the actual tissue assessment area. This problem would be solved by a new radiological technique, dual-energy CT (DECT). DECT can draw higher resolution images than conventional CT. The present study did not use DECT, as it is a newer method at Hirosaki University Hospital. This is a topic for future research.

In conclusion, the present study demonstrated that the TDC of dynamic CECT was associated with histological components of PDAC such as cancer cell density, CAF density, and microvessel density with quantitative methods and results for the first time. The initial slope of TDC was positively correlated with microvessel density, and the second slope of TDC was negatively correlated with cancer cell density and CAF 
density. The present study suggested that the TDC of dynamic CECT was useful to predict histological characteristics of PDAC.

\section{Acknowledgements}

The authors would like to thank Ms. Kana Saito, Ms. Shizuka Fujio, Mr. Yuta Yastuo and Mr. Takuya Shimanaka (Hirosaki University, Hirosaki, Japan) for providing technical assistance.

\section{Funding}

The present study was supported by JSPS Kakenhi (grant number JP19K16763).

\section{Availability of data and materials}

The datasets used and/or analyzed during the current study are available from the corresponding author on reasonable request.

\section{Authors' contributions}

SG and TY designed the experiments. SG performed the experiments and data analysis, wrote the main manuscript text and prepared the figures. HS evaluated the radiological images. SM and HK contributed in histological evaluation. $\mathrm{KI}$ and $\mathrm{KH}$ provided clinical information including adjuvant chemotherapy. TY and HK confirm the authenticity of all the raw data. All authors read and approved the manuscript, and agree to be accountable for all aspects of the research in ensuring that the accuracy or integrity of any part of the work are appropriately investigated and resolved.

\section{Ethics approval and consent to participate}

Human pancreatic ductal adenocarcinoma tissues were obtained with informed patient consent at the time of surgery at Hirosaki University Hospital (Hirosaki, Japan). This study was performed in accordance with the Declaration of Helsinki for Human Research and was approved by the Ethics Committee of Hirosaki University Graduate School of Medicine (protocol no. 2020-103).

\section{Patient consent for publication}

Not applicable.

\section{Competing interests}

The authors declare that they have no competing interests.

\section{References}

1. Stathis A and Moore MJ: Advanced pancreatic carcinoma: Current treatment and future challenges. Nat Rev Clin Oncol 7: 163-172, 2010

2. Bray F, Ferlay J, Soerjomataram I, Siegel RL, Torre LA and Jemal A: Global cancer statistics 2018: GLOBOCAN estimates of incidence and mortality worldwide for 36 cancers in 185 countries. CA Cancer J Clin 68: 394-424, 2018.

3. Ilic $\mathrm{M}$ and Ilic I: Epidemiology of pancreatic cancer. World J Gastroenterol 22: 9694-9705, 2016.
4. Feig C, Gopinathan A, Neesse A, Chan DS, Cook N and Tuveson DA: The pancreas histological characteristics. Clin Cancer Res 18: 4266-4276, 2012.

5. Kobayashi H, Enomoto A, Woods SL, Burt AD, Takahashi M and Worthley DL: Cancer-associated fibroblasts in gastrointestinal cancer. Nat Rev Gastroenterol Hepatol 16: 282-295, 2019.

6. Kalluri R: The biology and function of fibroblasts in cancer. Nat Rev Cancer 16: 582-598, 2016.

7. Neesse A, Bauer CA, Öhlund D, Lauth M, Buchholz M, Michl P, Tuveson DA and Gress TM: Stromal biology and therapy in pancreatic cancer: Ready for clinical translation? Gut 68: 159-171, 2019.

8. Öhlund D, Elyada E and Tuveson D: Fibroblast heterogeneity in the cancer wound. J Exp Med 211: 1503-1523, 2014.

9. Gieniec KA, Butler LM, Worthley DL and Woods SL: Cancer-associated fibroblasts-heroes or villains? Br J Cancer 121: 293-302, 2019.

10. Ishii G, Ochiai A and Neri S: Phenotypic and functional heterogeneity of cancer-associated fibroblast within the tumor microenvironment. Adv Drug Deliv Rev 99: 186-196, 2016.

11. Mezawa Y and Orimo A: The roles of tumor- and metastasis-promoting carcinoma-associated fibroblasts in human carcinomas. Cell Tissue Res 365: 675-689, 2016.

12. El Khouli RH, Macura KJ, Kamel IR, Jacobs MA and Bluemke DA: 3-T dynamic contrast-enhanced MRI of the breast: Pharmacokinetic parameters versus conventional kinetic curve analysis. AJR Am J Roentgenol 197: 1498-1505, 2011.

13. Yang SN, Li FJ, Chen JM, Zhang G, Liao YH and Huang TC: Kinetic curve type assessment for classification of breast lesions using dynamic contrast-enhanced MR imaging. PLoS One 11: e0152827, 2016.

14. Jabbar SB, Lynch B, Seiler S, Hwang H and Sahoo S: Pathologic findings of breast lesions detected on magnetic resonance imaging. Arch Pathol Lab Med 141: 1513-1522, 2017.

15. Hattori Y, Gabata T, Matsui O, Mochizuki K, Kitagawa H, Kayahara M, Ohta T and Nakanuma Y: Enhancement patterns of pancreatic adenocarcinoma on conventional dynamic multi-detector row CT: Correlation with angiogenesis and fibrosis. World J Gastroenterol 15: 3114-3121, 2009.

16. Brierley JD, Gospodarowicz MK and Wittekind C (eds): The TNM classification of malignant tumours, 8th edition. Wiley Blackwell, Oxford, pp93-95, 2017.

17. WHO Classification of Tumours Editorial Board. WHO classification of tumours of the digestive system. IARC Press, Lyon, pp322-332, 2019.

18. Inoue C, Miki Y, Saito R, Hata S, Abe J, Sato I, Okada Y and Sasano H: PD-L1 induction by cancer-associated fibroblast-derived factors in lung adenocarcinoma cells. Cancers (Basel) 11: 1257, 2019.

19. Itou RA, Uyama N, Hirota S, Kawada N, Wu S, Miyashita S, Nakamura I, Suzumura K, Sueoka H, Okada T, et al: Immunohistochemical characterization of cancer-associated fibroblasts at the primary sites and in the metastatic lymph nodes of human intrahepatic cholangiocarcinoma. Hum Pathol 83: 77-89, 2019.

20. Zhang J, Li S, Zhao Y, Ma P, Cao Y, Liu C, Zhang X, Wang W, Chen L and Li Y: Cancer-associated fibroblasts promote the migration and invasion of gastric cancer cells via activating IL-17a/JAK2/STAT3 signaling. Ann Transl Med 8: 877, 2020.

21. Amornsupak K, Jamjuntra P, Warnnissorn M, O-Charoenrat P, Sa-Nguanraksa D, Thuwajit P, Eccles SA and Thuwajit C: High $\mathrm{ASMA}^{+}$fibroblasts and low cytoplasmic $\mathrm{HMGB}^{+}$breast cancer cells predict poor prognosis. Clin Breast Cancer 17: 441-452.e2, 2017.

22. Matsuda K, Ohga N, Hida Y, Muraki C, Tsuchiya K, Kurosu T, Akino T, Shih SC, Totsuka Y, Klagsbrun M, et al: Isolated tumor endothelial cells maintain specific character during long-term culture. Biochem Biophys Res Commun 394: 947-954, 2010.

23. Yi CA, Lee KS, Kim EA, Han J, Kim H, Kwon OJ, Jeong YJ and Kim S: Solitary pulmonary nodules: Dynamic enhanced multi-detector row CT study and comparison with vascular endothelial growth factor and microvessel density. Radiology 233: 191-199, 2004.

24. Wang JH, Min PQ, Wang PJ, Cheng WX, Zhang XH, Wang Y, Zhao XH and Mao XQ: Dynamic CT evaluation of tumor vascularity in renal cell carcinoma. AJR Am J Roentgenol 186: 1423-1430, 2006.

25. El-Gohary YM, Metwally G, Saad RS, Robinson MJ, Mesko T and Poppiti RJ: Prognostic significance of intratumoral and peritumoral lymphatic density and blood vessel density in invasive breast carcinomas. Am J Clin Pathol 129: 578-586, 2008. 
26. Nieto Y, Woods J, Nawaz F, Baron A, Jones RB, Shpall EJ and Nawaz S: Prognostic analysis of tumour angiogenesis, determined by microvessel density and expression of vascular endothelial growth factor, in high-risk primary breast cancer patients treated with high-dose chemotherapy. Br J Cancer 97: 391-397, 2007.

27. Weidner N, Semple JP, Welch WR and Folkman J: Tumor angiogenesis and metastasis-correlation in invasive breast carcinoma. N Engl J Med 324: 1-8, 1991.

28. Macchiarini P, Fontanini G, Hardin MJ, Squartini F and Angeletti CA: Relation of neovascularisation to metastasis of nonsmall-cell lung cancer. Lancet 340: 145-146, 1992.

29. Fontanini G, Lucchi M, Vignati S, Mussi A, Ciardiello F, De Laurentiis M, De Placido S, Basolo F, Angeletti CA and Bevilacqua G: Angiogenesis as a prognostic indicator of survival in non-small-cell lung carcinoma: A prospective study. J Natl Cancer Inst 89: 881-886, 1997.

30. Vermeulen PB, Van den Eynden GG, Huget P, Goovaerts G, Weyler J, Lardon F, Van Marck E, Hubens G and Dirix LY: Prospective study of intratumoral microvessel density, p53 expression and survival in colorectal cancer. Br J Cancer 79: 316-322, 1999.

31. Katsuta E, Qi Q, Peng X, Hochwald SN, Yan L and Takabe K: Pancreatic adenocarcinomas with mature blood vessels have better overall survival. Sci Rep 9: 1310, 2019.

32. Awai $\mathrm{K}$ and Date S: Basic knowledge to achieve optimal enhancement of CT. Nichidoku Iho 56: 13-32, 2011.
33. Tsujino T, Seshimo I, Yamamoto H, Ngan CY, Ezumi K, Takemasa I, Ikeda M, Sekimoto M, Matsuura N and Monden M: Stromal myofibroblasts predict disease recurrence for colorectal cancer. Clin Cancer Res 13: 2082-2090, 2007.

34. Wang LM, Silva MA, D'Costa Z, Bockelmann R, Soonawalla Z, Liu S, O'Neill E, Mukherjee S, McKenna WG, Muschel R and Fokas E: The prognostic role of desmoplastic stroma in pancreatic ductal adenocarcinoma. Oncotarget 7: 4183-4194, 2016.

35. Miyai Y,EsakiN,Takahashi MandEnomoto A:Cancer-associated fibroblasts that restrain cancer progression: Hypotheses and perspectives. Cancer Sci 111: 1047-1057, 2020.

36. Alcaraz J, Carrasco JL, Millares L, Luis IC, Fernández-Porras FJ, Martínez-Romero A, Diaz-Valdivia N, De Cos JS, Rami-Porta R, Seijo L, et al: Stromal markers of activated tumor associated fibroblasts predict poor survival and are associated with necrosis in non-small cell lung cancer. Lung Cancer 135: 151-160, 2019.

37. Mizutani Y, Kobayashi H, Iida T, Asai N, Masamune A, Hara A, Esaki N, Ushida K, Mii S, Shiraki Y, et al: Meflin-positive cancer-associated fibroblasts inhibit pancreatic carcinogenesis. Cancer Res 79: 5367-5381, 2019.

(7) (9) This work is licensed under a Creative Commons Attribution-NonCommercial-NoDerivatives 4.0 International (CC BY-NC-ND 4.0) License. 